\title{
Targeting the SUMO pathway for neuroprotection in brain ischaemia
}

\author{
Wei Yang, ${ }^{1}$ Huaxin Sheng, ${ }^{1}$ Haichen Wang ${ }^{2}$
}

To cite: Yang W, Sheng $\mathrm{H}$ Wang H. Targeting the SUMO pathway for neuroprotection in brain ischaemia. Stroke and Vascular Neurology 2016;1:e00031.

doi:10.1136/svn-2016000031

Received 16 July 2016 Revised 5 August 2016 Accepted 6 August 2016 Published Online First 2 September 2016

\section{CrossMark}

\author{
${ }^{1}$ Multidisciplinary \\ Neuroprotection Laboratories, \\ Department of \\ Anesthesiology, Duke \\ University Medical Center, \\ Durham, North Carolina, USA \\ ${ }^{2}$ Multidisciplinary \\ Neuroprotection Laboratories, \\ Department of Neurology, \\ Duke University Medical \\ Center, Durham, North \\ Carolina, USA
}

Correspondence to Dr Wei Yang; wei.yang@duke.edu

\section{ABSTRACT}

Small ubiquitin-like modifier (SUMO) conjugation (SUMOylation) is a post-translational protein modification that modulates almost all major cellular processes, and has been implicated in many human diseases. A growing body of evidence from in vitro and in vivo studies demonstrates that increasing global levels of SUMO conjugated proteins (global SUMOylation) protects cells against ischaemia-induced damage, while suppressing global SUMOylation promotes cell injury after ischaemia. Indeed, SUMOylation has emerged as a potential therapeutic target for neuroprotection in brain ischaemia, including global brain ischaemia and focal brain ischaemia (ischaemic stroke). Here, we summarise findings on the role of SUMOylation in human diseases, brain ischaemia in particular, and review recent developments in drug discovery targeting SUMOylation with a major focus on its neuroprotective applications.

\section{INTRODUCTION}

The brain is particularly vulnerable to even a short period of insufficient blood supply, because of its lack of energy reserves. Ischaemic insult in the brain often impairs neurological functions, and is associated with major cardiac surgery and various pathological states of high clinical significance including cardiac arrest and ischaemic stroke. Each year, almost 800000 people in the USA alone suffer devastating consequences of stroke, ${ }^{1}$ and this problem is even more serious in developing countries. ${ }^{2}$ Stroke-induced disability is a great burden for patients and their families, and presents significant challenges for healthcare systems today. Since age is a key risk factor for stroke, these problems will become even more pronounced in the near future as the median age of our population continues to increase. Despite decades of efforts to develop strategies that protect the brain against ischaemia-induced damage, therapeutic hypothermia remains the only neuroprotective option in the clinic. No pharmacological neuroprotectant has yet been shown to improve outcomes for patients with brain ischaemia. Therefore, novel neuroprotective strategies are still urgently needed to increase resistance of the brain to ischaemic insult.

Brain ischaemia is a complex pathological process that involves many signalling pathways. Traditionally, single signalling pathways or functional processes have been explored as targets for therapeutic strategies that provide neuroprotection. However, all clinical trials based on this work have yielded disappointing results in the clinical setting. ${ }^{3} 4$ An approach that targets a multifunctional cellular process that coordinates many signalling pathways/processes in brain ischaemia may be a better strategy. ${ }^{4}$ Mounting evidence indicates that small ubiquitin-like modifier (SUMO) conjugation (SUMOylation), a posttranslational modification, is a promising candidate, and as such, a novel target with tremendous neuroprotective potential in brain ischaemia. In this review, we first provide a brief overview of the SUMO pathway, then summarise the current findings on the role of SUMOylation in human diseases, especially brain ischaemia, and finally highlight recent progress in drug discovery that targets global SUMOylation with a major focus on applications in brain iscahemia.

\section{The SUMO pathway}

SUMO is a group of small proteins consisting of about 100 amino acids. ${ }^{6}$ In mammalian cells, three SUMO isoforms have been discovered: SUMO1, 2 and 3. SUMO2 and SUMO3 are almost identical, and are thus normally referred to as SUMO2/3. SUMO1, however, shows only $50 \%$ sequence homology with SUMO2/3. It is important to note that despite their similarities, differences in expression levels of SUMO2 and SUMO3 can have a profound impact on physiological outcomes. For example, SUMO2 is the predominately expressed SUMO isoform in mice. Deletion of Sumo2 is embryonic lethal, while deletion of Sumo3 shows no overt phenotype. $^{7}$ 
SUMO proteins can covalently bond to the lysine residues of target proteins (SUMOylation), and thereby regulate their activity, stability and subcellular localisation. Before SUMOylation can occur, however, SUMO-specific proteases (SENPs) must proteolyticly process all SUMO precursors to expose the C-terminal functional motif. Similar to ubiquitination, SUMOylation requires three conserved steps that are catalysed by corresponding enzymes: an E1 activating enzymeSUMO-activating enzyme subunit $1 / 2$ (SAE1/SAE2), the solo E2 conjugating enzyme Ubc9, and various E3 ligating enzymes. The first activation step, mediated by an E1 enzyme, is an ATP-dependent reaction, and thus could be the limiting step of SUMOylation in certain conditions, including brain ischaemia, that cause rapid ATP depletion. Ubc9 is the only E2 conjugating enzyme that has been identified. Blocking or overexpressing Ubc9 has been widely used as an effective approach to study the consequence of modulating global SUMOylation in cells or animals under normal or stress conditions. ${ }^{8} 9$ The mechanism(s) that drive substrate specificity of SUMOylation are poorly understood. The interaction between substrates and the domains of specific E3 ligating enzymes contributes to the substrate selection in some cases. However, thousands of SUMO targets have been identified by proteomics studies, which is in sharp contrast to only a handful of known SUMO ligating enzymes. This suggests that many SUMO ligating enzymes have not yet been identified, and/or that other mechanisms could control the substrate specificity of SUMOylation.

SUMO modification is a dynamic process involving SUMOylation and de-SUMOylation. The de-SUMOylation to remove SUMO from targets is primarily mediated by SENPs. Six SENPs (SENP1-3 and 5-7) have been identified in mammalian cells (reviewed in Hickey et al). ${ }^{10}$ SENPs are cysteine proteases that play two major roles in the SUMO pathway: (1) as endopeptidases to remove the C-terminal extension and expose the di-glycine (GG) motif of SUMO precursors, and (2) as isopeptidases to de-conjugate SUMOylated proteins. SENPs show distinct SUMO preferences for endopeptidase and isopeptidase activities. Using purified proteins, Mendes $e t a l^{11}$ found that SENP1 and SENP2 are the most potent of the SENPs for endopeptidase and isopeptidase activity in all SUMO isoforms. However, studies on SENP mutant mice revealed that SENP1 acts to de-SUMOylate primarily SUMO1-conjugated proteins, while SENP2 preferentially de-conjugates SUMO2/ 3-conjugated proteins. ${ }^{12}{ }^{13}$ This in vivo information is crucial when targeting SENPs for drug discovery, since the compounds identified eventually will be tested in animals. For example, if the goal is to identify potent compounds that increase mainly the levels of SUMO2/ 3-conjugated proteins in animals, SENP2, among all SENPs, should be considered as a screening target. Of note, in addition to SENPs, three new SUMO proteases (DeSI-1, DeSI-2 and USPL1) have been identified. ${ }^{14} 15$
They appear to have de-conjugation capability on only a few particular substrates, however. Indeed, in contrast to SENPs, silencing these enzymes has no obvious effect on global SUMOylation in cells. ${ }^{14} 15$

\section{SUMOylation in human diseases}

SUMOylation regulates almost all major cellular processes including gene expression, DNA damage repair, RNA processing and quality control of newly synthesised proteins-all of which are essential for maintaining cellular homoeostasis. Numerous studies have now provided evidence that links SUMOylation to the pathophysiology of many diseases including cancer, heart diseases and brain ischaemia.

\section{Cancer}

Among all SUMO-related diseases investigated to date, cancer has been the most extensively studied. Considering the role of SUMOylation in maintaining cell function under stress/unfavourable conditions, it is not surprising that substantial evidence indicates a positive association between SUMOylation and cancer cell growth, tumourigenesis, metastasis and poor prognosis. ${ }^{16}$ For example, we found that levels of both Ubc9 and global SUMOylation are significantly increased in human brain tumour samples, and are most pronounced in glioblastoma multiforme tumours (GBM), the deadliest malignant primary brain tumours. ${ }^{17}$ Given that GBM are known for high resistance to standard radiotherapy and chemotherapy, and that SUMOylation is a key component in DNA damage repair processes, it is intriguing to speculate that inhibition of global SUMOylation may increase the sensitivity of glioblastomas to radiotherapy or chemotherapy. Importantly, Bossis $e t a l^{18}$ demonstrated that pharmacological inhibition of the SUMO pathway can overcome chemoresistance in some leukaemia cell lines. Targeting SUMOylation in cancer therapy has now attracted considerable interest. ${ }^{16}$

\section{Heart diseases}

SUMO homoeostasis must be finely balanced for normal heart development and function. An imbalance in SUMOylation/de-SUMOylation contributes to cardiovascular heart diseases (for detailed review, see Mendler et $a l) .{ }^{19}$ For example, SUMOylation of the sarcoplasmic/endoplasmic reticulum (ER) $\mathrm{Ca}^{2+}$ ATPase (SERCA2a) positively controls its activity and stability, which is critical for heart function. In mice with heart failure, the levels of SUMO1 decrease in the heart. Consequently, SERCA2a loses its SUMOylation state, which reduces its ATP-binding affinity and ATPase activity. Notably, the pathological state of the failing heart can be substantially alleviated by virus-mediated SUMO1 gene delivery. ${ }^{20}$ Similarly, in a swine model of ischaemic heart failure, viral transfer of the SUMO1 gene significantly improves cardiac function. ${ }^{21}$ Although these studies indicate that SUMOylation-based strategies have 
great potential for treating heart diseases, published findings in this field are not consistent, and thus a better understanding of the role of SUMOylation in heart diseases is still needed. ${ }^{19}$

\section{Brain ischaemia}

A massive increase in global SUMOylation in the brain was first observed in hibernating squirrels. ${ }^{8}$ In this study, the Hallenbeck group used hibernation as a natural ischaemia tolerance model to identify endogenous neuroprotective pathways, and found a marked increase in the levels of both SUMO1-conjugated and SUMO2/ 3-conjugated proteins in the brain during the torpor phase of hibernation. During torpor, the body temperature is $\sim 5^{\circ} \mathrm{C}$, and heart rate and cerebral blood flow are dramatically reduced to otherwise lethal levels. The authors therefore hypothesised that increased global SUMOylation is a molecular mechanism underlying this ischaemia tolerance during torpor, that is, hypothermiamediated neuroprotection. To support this hypothesis, they showed that preconditioning (a short period of oxygen/glucose deprivation (OGD)) with hypothermia increases global SUMOylation in SHSY5Y cells, which in turn, contributes to cell protection against severe OGD, an in vitro ischaemia model. Since therapeutic hypothermia is currently used in the clinic to protect the brain from ischaemic damage, our group set out to determine whether SUMOylation also plays a role in neuroprotection conferred by therapeutic hypothermia. We demonstrated, for the first time, that when animals are exposed to various degrees of hypothermia ranging from $18^{\circ} \mathrm{C}$ to $30^{\circ} \mathrm{C}$, both deep and moderate hypothermia activate the SUMOylation pathway, and induce nuclear accumulation of SUMO2/3-conjugated proteins. ${ }^{22}{ }^{23}$ Therefore, we proposed that SUMOylation contributes to the neuroprotection afforded by therapeutic hypothermia. This notion has been substantiated by the finding that hypothermic treatment has no additional protective effect on stroke outcome in Ubc9 transgenic mice, which sustain high levels of global SUMOylation. ${ }^{24}$ Together, these results strongly support a major role for increased global SUMOylation in hypothermia-mediated brain protection against ischaemic insults. However, to definitively prove this hypothesis, genetically modified SUMO mice and pharmacological modulators of the SUMOylation pathway may be required in future studies. If this hypothesis is eventually validated, pharmacologically increasing global SUMOylation could be a promising clinical strategy for protecting the brain against ischaemic damage associated with various surgical procedures, and thereby avoid the adverse effects related to therapeutic hypothermia treatment.

The first evidence that established a link between SUMOylation and a pathological state of brain ischaemia was reported by our group. We found that transient forebrain ischaemia rapidly induces a massive increase in the levels of SUMO2/3-conjugated proteins during reperfusion. ${ }^{25}$ This finding initiated many follow-up studies on SUMOylation in various brain ischaemia animal models. ${ }^{9} 24{ }^{26-30}$ In one of these studies, we showed that after transient focal cerebral ischaemia induced by middle cerebral artery occlusion (MCAO), an ischaemic stroke model, activation of SUMO2/3 conjugation is most pronounced in neurons located at the border of the tissue supplied by the MCA where it is characterised by translocation of SUMO-conjugated proteins to the nucleus. Surprisingly, even a short, nondamaging period of MCA occlusion is sufficient to massively activate this process. ${ }^{27}$ These data suggest that postischaemic activation of $\mathrm{SUMO} / 3$ conjugation is a protective stress response in neurons. This notion is corroborated by results from cell culture and animal studies. ${ }^{8} 9$ 31-33 For example, under normal conditions, blocking SUMO2/3 conjugation in primary neurons does not trigger cell damage. However, after OGD, it significantly increases cell death. ${ }^{33}$ Transgenic mice overexpressing Ubc9 (the only SUMO E2 conjugating enzyme) exhibit increased levels of SUMO1-conjugated and SUMO2/3-conjugated proteins, and show improved outcome after stroke. ${ }^{9}$ These data demonstrate the neuroprotective role of SUMO conjugation after brain ischaemia. To more directly characterise the function of each SUMO in brain ischaemia, individual SUMO knockout mice are needed. All of these mice have recently become available, ${ }^{7} 34$ except SUMO2 conditional knockout mice. ${ }^{7}$

\section{Potential mechanisms underlying SUMOylation-mediated neuroprotection in brain ischaemia}

Thousands of SUMO target proteins have been identified. The increase in global SUMOylation observed in brain ischaemia is expected to affect many proteins and their related pathways/processes. Thus, it could be difficult, if not impossible, to pinpoint one, or even a few SUMO targets or pathways that play a key role in SUMOylation-mediated neuroprotection. However, recent progress in this field provided valuable information about potential mechanisms, including crosstalk between SUMOylation and ubiquitination, and the change in SUMOylation status of proteins that are crucial to the pathogenesis of brain ischaemia.

The crosstalk between SUMOylation and ubiquitination has been reported in several cell culture proteomics studies. One study demonstrated that SUMOylationactivated ubiquitination is essential for quality control of newly synthesised proteins and misfolded proteins. ${ }^{35}$ Interestingly, the accumulation of misfolded proteins in the ER causes ER stress, which is a hallmark of brain ischaemia. ER stress-mediated cell death is a major factor in ischaemic brain damage. Therefore, SUMOylation-dependent ubiquitination may be involved in defining the fate and function of postischaemic cells. Indeed, a recent proteomics study, using a novel SUMO transgenic mouse model with forebrain-specific expression of epitope-tagged SUMO1-3, revealed, for the first time, the extensive in vivo crosstalk between 
ubiquitination and SUMOylation after transient forebrain ischaemia. $^{26}$

Identification and characterisation of individual SUMO targets involved in brain ischaemia is just beginning. Many important proteins in ischaemia-related signalling pathways and neuronal function are SUMO targets. These include hypoxia inducible factor 1 , heat shock factor $1, \mathrm{I} \kappa \mathrm{B} \alpha$ and potassium channel proteins. ${ }^{36}$ However, the extent to which SUMOylation of these proteins is involved in the response to brain ischaemia is largely unknown. A recent proteomics study on brain samples collected after forebrain ischaemia, has generated the first data set of in vivo SUMO targets regulated by ischaemia. This data set presents a valuable resource for future studies. In this study, western blot analysis showed that glucocorticoid receptor (GR) is the most prominent SUMO target induced by ischaemia. This is an important discovery and warrants further study, because SUMOylation critically modulates GR activity, and the GR pathway contributes to brain ischaemia outcome. To date, only two studies have reported findings on the role of SUMOylation of a particular protein in cell death after ischaemic stress. In an in vitro OGD study, Guo et $a l^{37}$ reported that SUMOylation of Drp1 ensures that Drp1 remains in the cytoplasm and thus, suppresses Drp1-mediated release of cytochrome c from mitochondria, thereby preventing cell death. A recent in vivo MCAO study provided some evidence suggesting that NCX3 SUMOylation is involved in SUMO-mediated beneficial effects during ischaemic preconditioning. ${ }^{28}$

\section{Drug discovery targeting SUMOylation for neuroprotection}

As discussed above, an increase in global SUMOylation is believed to be an endogenous neuroprotective mechanism whereby brain cells become more resistant to a transient episode of low blood flow. Considering the multifactorial effects of SUMOylation, and the continuing failure of clinic trials that target a single pathway/ process to neuroprotection in stroke, we believe that SUMOylation is a promising therapeutic target for brain ischaemia. In fact, while the protective mechanisms of SUMOylation in brain ischaemia continue to be unravelled, considerable effort has already been directed towards identifying compounds that pharmacologically increase global SUMOylation, and testing the feasibility of this strategy for neuroprotection.

In contrast to the many years of research on drug discovery in the ubiquitin field, ${ }^{38}$ targeting SUMOylation for therapeutic purposes is still in its infancy. SUMOylation and ubiquitination are highly homologous as they both require three enzymatic steps to conjugate their respective modifiers to lysine residues on target proteins, and one enzymatic step for de-conjugation. Therefore, advances in the ubiquitin field will inform the development of potential SUMOylation-based therapeutic interventions.

In 2003, the Food and Drugs Administration (FDA) approved bortezomib (Velcade, Millennium
Pharmaceuticals), the first successful anticancer drug that targets the ubiquitin-proteasome system. $^{38}$ The implications of this approval are significant because it not only confirms that the ubiquitin pathway is a valid drug target, but it also demonstrates that targeting a complex network that modulates many proteins is a feasible approach. Recently, de-ubiquitylating enzymes (DUBs), which are critical regulators of the ubiquitinproteasome system, have emerged as potential drug targets. ${ }^{39}$ Many drug discovery studies that target the isopeptidase activity of DUBs, have reported impressive results. ${ }^{39}$ Further, Reverdy et $a l^{40}$ have shown that it is feasible to identify compounds with high selectivity for a specific DUB. In the SUMO pathway, SENPs are the counterparts of DUBs.

The remarkable progress in the ubiquitin field has inspired confidence that SUMOylation is also a promising target for drug discovery. Since boosting global SUMOylation is believed to be neuroprotective in brain ischaemia, we summarise here only the recent discoveries of compounds that increase SUMOylation. Increasing global SUMOylation requires balance between conjugation and de-conjugation processes. Thus, compounds that activate the SUMO E1 or E2 enzyme, or inhibit SENP de-conjugation enzymes are expected to achieve this goal. A recent study used SUMO E1 and E2 as targets to screen over 100000 compounds from large National Cancer Institute (NCI) libraries. ${ }^{41}$ The most promising compound identified was N106, which can increase SUMO conjugation in cells through direct activation of the SUMO E1 enzyme. Notably, treatment with N106 shows beneficial effects on cardiac function in a mouse model of heart failure. If this compound is blood-brain barrier permeable, it may also be applicable in brain ischaemia.

However, it is generally believed that inhibition of enzymes by small molecules is much less challenging than activation. Therefore, perhaps the best strategy to identify new classes of compounds that increase SUMOylation is to search for compounds that block de-SUMOylation by inhibiting the isopeptidase activity of SENPs. Indeed, major efforts in this field have been put into screening for specific SENP inhibitors. To date, several classes of SENP inhibitors have been discovered (table 1). Of note, in most of those studies, SENP1 has been used as the screening target due to its preference for SUMO1-conjugated proteins and its key role in cancer development.

Notably, only two virtual screening studies provide evidence that the inhibitors identified can increase global SUMOylation in cells, ${ }^{44}{ }^{46}$ and their effectiveness in animals is not yet known. Further, most SENP inhibitors reported to date appear to be suitable only as analytical tools because they lack drug-like properties. Thus, there is still a great need to identify new classes of specific SENP inhibitors. For this, conventional high throughput screening (HTS) is more promising than virtual screening. Currently, there are a few biochemical assays that can 
Table 1 Summary of SENP inhibitors identified by screening

\begin{tabular}{|c|c|c|c|c|c|c|}
\hline $\begin{array}{l}\text { Screening } \\
\text { target }\end{array}$ & Screening strategy & $\begin{array}{l}\text { Screening } \\
\text { library }\end{array}$ & $\begin{array}{l}\text { Representative } \\
\text { hit }\end{array}$ & $I_{50}$ & $\begin{array}{l}\text { Bioactivity } \\
\text { in cells }\end{array}$ & Reference \\
\hline SENP1 & Benzodiazepine-based design & - & Compound 38 & $9.2 \mu \mathrm{M}$ & Not tested & 42 \\
\hline SENP1 & $\begin{array}{l}\text { Cleavage of fluorogenic } \\
\text { substrate SUMO1-AMC }\end{array}$ & $\begin{array}{l}\text { In-house } \\
\text { compound library }\end{array}$ & GN6958 & $29.6 \mu \mathrm{M}$ & Not tested & 43 \\
\hline SENP1 & Virtual screening & SPECS database & $\mathrm{SI}$ & $1.29 \mu \mathrm{M}$ & Confirmed & 44 \\
\hline PfSENP1 & $\begin{array}{l}\text { SUMO-procleavage assay } \\
\text { with SDS-PAGE detection }\end{array}$ & $\begin{array}{l}\text { Cysteine } \\
\text { protease } \\
\text { inhibitors library }\end{array}$ & JCP-666 & $17.9 \mu \mathrm{M}$ & Not tested & 45 \\
\hline SENP1/2 & Virtual screening & DTP & NSC5068 & $\begin{array}{l}\mu \mathrm{M} \\
\text { range }\end{array}$ & Confirmed & 46 \\
\hline SENP2 & $\begin{array}{l}\text { Combination of virtual } \\
\text { screening and FRET-based } \\
\text { assay }\end{array}$ & $\begin{array}{l}\text { Namiki-shoji } \\
\text { library }\end{array}$ & 1,2,5-oxadiazoles & $<10 \mu \mathrm{M}$ & Not tested & 47 \\
\hline SENPS & $\begin{array}{l}\text { Cleavage of fluorogenic } \\
\text { AFC-based substrates }\end{array}$ & $\begin{array}{l}\text { In-house } \\
\text { compound library }\end{array}$ & VEA499 & $\begin{array}{l}\mu \mathrm{M} \\
\text { range }\end{array}$ & No activity & 48 \\
\hline
\end{tabular}

be adapted to screen for SENP inhibitors in an HTS system. ${ }^{49}$ Most of these assays use an SENP catalytic fragment and artificial peptide-based fluorogenic substrates such as SUMO1-7-amino-4-methylcoumarin (AMC), because this kind of assay is easy to set up, and the fluorescence signal generated by a leaving group, for example, AMC, can be quantitatively measured. Fluorescence resonance energy transfer (FRET)-based assays have also been developed. The substrate used in these assays is a SUMO precursor flanked by two fluorescent proteins, which produce a FRET signal if the substrate is intact and not cleaved by SENP. A few HTS studies that used these assay methods have been carried out, and the data are deposited in the PubChem BioAssay database (http://pubchem.ncbi.nlm.nih.gov/). Surprisingly, no hits from these screens have yet been reported in research publications.

Here, we propose that physiologically relevant assay substrates, that is, SUMO-conjugated proteins, be used to search for SENP inhibitors in future HTS projects, rather than the artificial substrates aforementioned. Indeed, the data from a nuclear magnetic resonance analysis of the inhibition mechanism of a compound indicated that the inhibitory effect could vary depending on whether an artificial substrate or a physiological substrate is used. ${ }^{46}$ Although two groups have established FRET-based assays using a physiological substrate-SUMO1-conjugated RanGAP1 - neither assay is designed for HTS. ${ }^{50} 51$ Our group has developed an AlphaScreen-based and HTS-compatible assay that is designed to screen for inhibitors of the isopeptidase activity of SENP. ${ }^{52}$ This assay uses a SUMO-conjugated protein as substrate, and is robust and reliable. ${ }^{49}$ This platform, therefore, can be used in future HTS studies to screen for new classes of inhibitors targeting SENP isopeptidase activity. Such future efforts may have a great impact on research in neuroprotection because the new drug-like SENP inhibitors identified are expected to have great potential to boost global SUMOylation in the brain, thereby increasing the brain's resistance to ischaemic injury, and improving outcomes following an ischaemic event.

Of note, a recent study took a different approach to increase SUMOylation, and identified specific inhibitors of miR-182 or miR-183. ${ }^{53}$ The rationale for this study is that levels of the miR-200 family and the miR-182 family decrease in squirrel brain during the torpor phase when global SUMOylation is increased, and further, that inhibiting these microRNAs increases global SUMOylation in cells. ${ }^{53}$ In this screening, the authors designed a luciferase reporter assay, and screened three small libraries. A dozen compounds were identified, and interestingly, most of them could increase global SUMOylation and provide protective effects for cells in OGD experiments. Since the screening libraries used are collections of pharmacologically active compounds that are in clinical use, clinical testing or preclinical testing, the repurposing potential of these compounds in animal models of brain ischaemia is high.

\section{Conclusions and perspectives}

A substantial amount of evidence now supports the notion that increased global SUMOylation is protective in postischaemic cells. Notably, a recent study showed that the flavonoid quercetin protects cells against OGD. This protection is largely mediated by increasing global SUMOylation via the inhibitory effect of quercetin on SENPs. ${ }^{54}$ This study by Lee and colleagues demonstrated, for the first time, that increasing global SUMOylation via an SENP inhibitor is neuroprotective in cell culture. Taken together, we believe that the research in this field has laid the foundation for launching drug discovery efforts that target SUMOylation to promote neuroprotection in brain ischaemia. This will require a close collaboration between experimental 
neurology groups and drug discovery groups. Future HTS projects are expected to use physiologically relevant substrates to screen a variety of large libraries. Further, it may be best to search for pan SENP inhibitors rather than SENP-specific inhibitors, in order to increase the probability of success in testing this novel neuroprotective strategy in brain ischaemia. Therefore, the catalytic C-terminal fragment of an SENP, which contains the conserved cysteine protease domain, should be used as the screening target rather than a full-length SENP protein because data suggest that in cells, the N-terminal regions of SENPs determine substrate specificity. ${ }^{55}$ As more efforts are invested in this field, we expect that data from a proof-of-concept animal experiment will become available soon, and will validate this new exciting target for providing neuroprotection in the brain that is under ischaemic insult.

\section{Acknowledgements The authors thank Kathy Gage for her excellent editorial contributions.}

Contributors WY conceived and drafted the review with critical input from HS and HW.

Funding This study was partly supported by American Heart Association grant number 12SDG11950003 and National Institutes of Health (NIH) grant number NS081299.

Competing interests None declared.

Provenance and peer review Not commissioned; externally peer reviewed.

Data sharing statement No additional data are available.

Open Access This is an Open Access article distributed in accordance with the Creative Commons Attribution Non Commercial (CC BY-NC 4.0) license, which permits others to distribute, remix, adapt, build upon this work noncommercially, and license their derivative works on different terms, provided the original work is properly cited and the use is non-commercial. See: http:// creativecommons.org/licenses/by-nc/4.0/

\section{REFERENCES}

1. Mozaffarian D, Benjamin EJ, Go AS, et al. Heart Disease and Stroke Statistics-2016 update: a report from the American Heart Association. Circulation 2016;133:e38-e360.

2. Wang Y, Wang D. Hand in hand with the world to conquer stroke. Stroke Vasc Neurol 2016;1:e000007.

3. Moretti A, Ferrari F, Villa RF. Neuroprotection for ischaemic stroke: current status and challenges. Pharmacol Ther 2015;146:23-34.

4. Savitz SI, Fisher M. Future of neuroprotection for acute stroke: in the aftermath of the SAINT trials. Ann Neurol 2007;61:396-402.

5. Yang W, Sheng H, Homi HM, et al. Cerebral ischemia/stroke and small ubiquitin-like modifier (SUMO) conjugation-a new target for therapeutic intervention? J Neurochem 2008;106:989-99.

6. Yang W, Paschen W. SUMO proteomics to decipher the SUMO-modified proteome regulated by various diseases. Proteomics 2015;15:1181-91.

7. Wang L, Wansleeben C, Zhao S, et al. SUMO2 is essential while SUMO3 is dispensable for mouse embryonic development. EMBO Rep 2014:15:878-85.

8. Lee YJ, Miyake $\mathrm{S}$, Wakita $\mathrm{H}$, et al. Protein SUMOylation is massively increased in hibernation torpor and is critical for the cytoprotection provided by ischemic preconditioning and hypothermia in SHSY5Y cells. J Cereb Blood Flow Metab 2007;27:950-62.

9. Lee YJ, Mou Y, Maric D, et al. Elevated global SUMOylation in Ubc9 transgenic mice protects their brains against focal cerebral ischemic damage. PLOS ONE 2011;6:e25852.

10. Hickey CM, Wilson NR, Hochstrasser M. Function and regulation of SUMO proteases. Nat Rev Mol Cell Biol 2012;13:755-66.

11. Mendes AV, Grou CP, Azevedo JE, et al. Evaluation of the activity and substrate specificity of the human SENP family of SUMO proteases. Biochim Biophys Acta 2016;1863:139-47.
12. Sharma P, Yamada S, Lualdi M, et al. Senp1 is essential for desumoylating Sumo1-modified proteins but dispensable for Sumo2 and Sumo3 deconjugation in the mouse embryo. Cell Rep 2013:3:1640-50.

13. Kang X, Qi Y, Zuo Y, et al. SUMO-specific protease 2 is essential for suppression of polycomb group protein-mediated gene silencing during embryonic development. Mol Cell 2010;38:191-201.

14. Shin EJ, Shin HM, Nam E, et al. DeSUMOylating isopeptidase: a second class of SUMO protease. EMBO Rep 2012;13:339-46.

15. Schulz S, Chachami G, Kozaczkiewicz L, et al. Ubiquitin-specific protease-like 1 (USPL1) is a SUMO isopeptidase with essential, non-catalytic functions. EMBO Rep 2012;13:930-8.

16. Eifler K, Vertegaal AC. SUMOylation-mediated regulation of cell cycle progression and cancer. Trends Biochem Sci 2015;40:779-93.

17. Yang W, Wang L, Roehn G, et al. Small ubiquitin-like modifier 1-3 conjugation [corrected] is activated in human astrocytic brain tumors and is required for glioblastoma cell survival. Cancer $\mathrm{Sci}$ 2013;104:70-7.

18. Bossis G, Sarry JE, Kifagi C, et al. The ROS/SUMO axis contributes to the response of acute myeloid leukemia cells to chemotherapeutic drugs. Cell Rep 2014;7:1815-23.

19. Mendler L, Braun T, Muller S. The ubiquitin-like SUMO system and heart function: from development to disease. Circ Res 2016;118:132-44.

20. Kho C, Lee A, Jeong D, et al. SUMO1-dependent modulation of SERCA2a in heart failure. Nature 2011:477:601-5.

21. Tilemann L, Lee $A$, Ishikawa $K$, et al. SUMO-1 gene transfer improves cardiac function in a large-animal model of heart failure. Sci Transl Med 2013;5:211ra159.

22. Yang W, Ma Q, Mackensen GB, et al. Deep hypothermia markedly activates the small ubiquitin-like modifier conjugation pathway; implications for the fate of cells exposed to transient deep hypothermic cardiopulmonary bypass. J Cereb Blood Flow Metab 2009;29:886-90.

23. Wang L, Ma Q, Yang $\mathrm{W}$, et al. Moderate hypothermia induces marked increase in levels and nuclear accumulation of SUMO2/ 3-conjugated proteins in neurons. J Neurochem 2012;123:349-59.

24. Lee YJ, Mou Y, Klimanis D, et al. Global SUMOylation is a molecular mechanism underlying hypothermia-induced ischemic tolerance. Front Cell Neurosci 2014;8:416.

25. Yang W, Sheng H, Warner DS, et al. Transient global cerebral ischemia induces a massive increase in protein sumoylation. $J$ Cereb Blood Flow Metab 2008;28:269-79.

26. Yang W, Sheng $\mathrm{H}$, Thompson JW, et al. Small ubiquitin-like modifier 3-modified proteome regulated by brain ischemia in novel small ubiquitin-like modifier transgenic mice: putative protective proteins/ pathways. Stroke 2014;45:1115-22.

27. Yang W, Sheng H, Warner DS, et al. Transient focal cerebral ischemia induces a dramatic activation of small ubiquitin-like modifier conjugation. J Cereb Blood Flow Metab 2008;28:892-6.

28. Cuomo O, Pignataro G, Sirabella R, et al. Sumoylation of LYS590 of NCX3 f-Loop by SUMO1 participates in brain neuroprotection induced by ischemic preconditioning. Stroke 2016;47:1085-93.

29. Hochrainer K, Jackman K, Benakis C, et al. SUMO2/3 is associated with ubiquitinated protein aggregates in the mouse neocortex after middle cerebral artery occlusion. J Cereb Blood Flow Metab 2015;35:1-5.

30. Cimarosti $\mathrm{H}$, Lindberg $\mathrm{C}$, Bomholt SF, et al. Increased protein SUMOylation following focal cerebral ischemia. Neuropharmacology 2008;54:280-9.

31. Lee YJ, Castri P, Bembry J, et al. SUMOylation participates in induction of ischemic tolerance. J Neurochem 2009;109:257-67.

32. Cimarosti $\mathrm{H}$, Ashikaga $\mathrm{E}$, Jaafari $\mathrm{N}$, et al. Enhanced SUMOylation and SENP-1 protein levels following oxygen and glucose deprivation in neurones. J Cereb Blood Flow Metab 2012;32:17-22.

33. Datwyler AL, Lattig-Tunnemann G, Yang W, et al. SUMO2/3 conjugation is an endogenous neuroprotective mechanism. $J$ Cereb Blood Flow Metab 2011;31:2152-9.

34. Evdokimov E, Sharma P, Lockett SJ, et al. Loss of SUMO1 in mice affects RanGAP1 localization and formation of PML nuclear bodies, but is not lethal as it can be compensated by SUMO2 or SUMO3. $J$ Cell Sci 2008;121:4106-13.

35. Tatham MH, Matic I, Mann M, et al. Comparative proteomic analysis identifies a role for SUMO in protein quality control. Sci Signal 2011;4:rs4.

36. Silveirinha V, Stephens GJ, Cimarosti H. Molecular targets underlying SUMO-mediated neuroprotection in brain ischemia. J Neurochem 2013;127:580-91.

37. Guo C, Hildick KL, Luo J, et al. SENP3-mediated deSUMOylation of dynamin-related protein 1 promotes cell death following ischaemia. EMBO J 2013;32:1514-28. 
38. Bedford L, Lowe J, Dick LR, et al. Ubiquitin-like protein conjugation and the ubiquitin-proteasome system as drug targets. Nat Rev Drug Discov 2011;10:29-46.

39. Pal A, Young MA, Donato NJ. Emerging potential of therapeutic targeting of ubiquitin-specific proteases in the treatment of cancer. Cancer Res 2014;74:4955-66.

40. Reverdy C, Conrath S, Lopez R, et al. Discovery of specific inhibitors of human USP7/HAUSP deubiquitinating enzyme. Chem Biol 2012;19:467-77.

41. Kho C, Lee A, Jeong D, et al. Small-molecule activation of SERCA2a SUMOylation for the treatment of heart failure. Nat Commun 2015;6:7229.

42. Qiao Z, Wang W, Wang L, et al. Design, synthesis, and biological evaluation of benzodiazepine-based SUMO-specific protease 1 inhibitors. Bioorg Med Chem Lett 2011;21:6389-92.

43. Uno M, Koma Y, Ban HS, et al. Discovery of 1-[4-(N-benzylamino) phenyl]-3-phenylurea derivatives as non-peptidic selective SUMO-sentrin specific protease (SENP)1 inhibitors. Bioorg Med Chem Lett 2012;22:5169-73.

44. Wen D, Xu Z, Xia L, et al. Important role of SUMOylation of spliceosome factors in prostate cancer cells. J Proteome Res 2014;13:3571-82

45. Ponder EL, Albrow VE, Leader BA, et al. Functional characterization of a SUMO deconjugating protease of Plasmodium falciparum using newly identified small molecule inhibitors. Chem Biol 2011:18:711-21.

46. Madu IG, Namanja AT, Su Y, et al. Identification and characterization of a new chemotype of noncovalent SENP inhibitors. ACS Chem Biol 2013;8:1435-41.
47. Kumar A, Ito A, Takemoto M, et al. Identification of 1,2,5-oxadiazoles as a new class of SENP2 inhibitors using structure based virtual screening. J Chem Inf Model 2014;54:870-80.

48. Albrow VE, Ponder EL, Fasci D, et al. Development of small molecule inhibitors and probes of human SUMO deconjugating proteases. Chem Biol 2011;18:722-32.

49. Madu IG, Chen Y. Assays for investigating deSUMOylation enzymes. Curr Protoc Mol Biol 2012;99:10.30.1-10.30.13.

50. Stankovic-Valentin N, Kozaczkiewicz L, Curth K, et al. An in vitro FRET-based assay for the analysis of SUMO conjugation and isopeptidase cleavage. Methods Mol Biol 2009;497:241-51.

51. Tatham MH, Hay RT. FRET-based in vitro assays for the analysis of SUMO protease activities. Methods Mol Biol 2009;497:253-68.

52. Yang W, Wang L, Paschen W. Development of a high-throughput screening assay for inhibitors of small ubiquitin-like modifier proteases. J Biomol Screen 2013;18:621-8.

53. Lee YJ, Johnson KR, Hallenbeck JM. Global protein conjugation by ubiquitin-like-modifiers during ischemic stress is regulated by microRNAs and confers robust tolerance to ischemia. PLOS ONE 2012;7:e47787.

54. Lee YJ, Bernstock JD, Nagaraja N, et al. Global SUMOylation facilitates the multimodal neuroprotection afforded by quercetin against the deleterious effects of oxygen/glucose deprivation and the restoration of oxygen/glucose. $J$ Neurochem 2016:138:101-16.

55. Kolli N, Mikolajczyk J, Drag M, et al. Distribution and paralogue specificity of mammalian deSUMOylating enzymes. Biochem $J$ 2010;430:335-44. 